\title{
Ocorrência de Vampyressa pusilla (Chiroptera, Phyllostomidae) no Pantanal sul
}

\author{
José Milton Longo ${ }^{1}$, Erich Fischer ${ }^{2,4}$, George Camargo ${ }^{3}$ \& Carolina Ferreira Santos ${ }^{1}$ \\ Biota Neotropica $v 7(n 3)$ \\ http://www.biotaneotropica.org.br/v7n3/pt/abstract?short-communication+bn02407032007 \\ Recebido em 29/03/07 \\ Versão Reformulada recebida em 31/08/07 \\ Publicado em 28/09/07 Universidade Federal de Mato Grosso do Sul, CEP 79070-900, Campo Grande, Brasil, http://www.dbi.ufms.br/mstecopan/ecologia.htm ${ }^{2}$ Departamento de Biologia, Universidade Federal de Mato Grosso do Sul - UFMS CP 549, CEP 79070-900, Campo Grande, Brasil, http://www.ufms.br, ${ }^{3}$ Conservação Internacional do Brasil, Rua Paraná, 32, CEP 79021-220, Campo Grande, Mato Grosso do Sul, Brasil, http://www.conservation.org.br, ${ }^{4}$ Autor para correspondência: Erich Fischer,e-mail: eafischer@uol.com.br \\ ${ }^{1}$ Programa de Pós-Graduação em Ecologia e Conservação, Centro de Ciências Biológicas e da Saúde,
}

\begin{abstract}
Longo, J.M., Fischer, E., Camargo, G. \& Santos, C.F. Occurrence of Vampyressa pusilla (Chiroptera, Phyllostomidae) in Southern Pantanal. Biota Neotrop. Sep/Dez 2007 vol. 7, no. 3 http://www.biotaneotropica. org.br/v7n3/pt/abstract?short-communication+bn02407032007. ISSN 1676-0603.

Species of Vampyressa Thomas, 1900 are locally uncommon and widespread in the Neotropics. They are known to occur from southern Mexico to southern Amazon basin and from southeastern South America to Paraguay. Vampyressa pusilla (Wagner, 1843) and V. thyone Thomas, 1909 - previously considered one species - show a disjointed distribution. The former is considered endemic to the Atlantic forest and the other occurs from northwestern South America to southern Mexico. In addition, V. pusilla has been registered in savanna (Cerrado) and dry forests (Chaco). We report here the occurrence of $V$. pusilla in the Pantanal wetlands, western Brazil. It is probably the first record of the genus Vampyressa in such an ecosystem, increasing the geographical range of $V$. pusilla to western Brazil, towards the V. thyone distribution limits.

Keywords: bats, Brazil, Chiroptera, geographic range, Nhecolândia, wetlands.

\section{Resumo}

Longo, J.M., Fischer, E., Camargo, G. \& Santos, C.F. Ocorrência de Vampyressa pusilla (Chiroptera, Phyllostomidae) no Pantanal sul. Biota Neotrop. Sep/Dez 2007 vol. 7, no. 3 http://www.biotaneotropica.org. br/v7n3/pt/abstract?short-communication+bn02407032007. ISSN 1676-0603.

Espécies de Vampyressa Thomas, 1900, são localmente raras e amplamente distribuídas nos Neotrópicos. Ocorrem desde o sul do México até o sul da bacia Amazônica e do sudeste da América do Sul até o Paraguai. Vampyressa pusilla (Wagner, 1843) e V. thyone Thomas, 1909 - anteriormente consideradas uma espécie - apresentam distribuição disjunta, a primeira é considerada endêmica da Mata Atlântica e a segunda ocorre do noroeste da América do Sul ao sul do México. Adicionalmente, V. pusilla tem sido registrada em Cerrado e florestas secas (Chaco). Reportamos aqui a ocorrência de V. pusilla na planície inundável do Pantanal, oeste do Brasil. Provavelmente este é o primeiro registro do gênero Vampyressa neste ecossistema, ampliando a distribuição geográfica de $V$. pusilla para o oeste brasileiro, em direção aos limites de distribuição de $V$. thyone.
\end{abstract}

Palavras-chave: Brasil, Chiroptera, distribuição geográfica, morcegos, Nhecolândia, planície inundável. 


\section{Introdução}

O gênero Vampyressa Thomas, 1900 (Phyllostomidae) compreende espécies de morcegos frugívoros que utilizam principalmente figos em sua dieta (Bonaccorso 1979, Pedro et al. 1997). Espécies de Vampyressa são localmente raras e amplamente distribuídas, ocorrendo desde o sul do México até o sul do Peru, assim como na bacia Amazônica, sudeste da América do Sul e sul do Paraguai (Lewis \& Wilson 1987, Miretzki 2003). As espécies de Vampyressa e de alguns outros gêneros de morcegos neotropicais apresentam distribuição geográfica disjunta devido à provável não ocorrência em formações áridas na região central da América do Sul (Lewis \& Wilson 1987, Camargo \& Fischer 2005). A ausência de registros no Pantanal determina a separação das distribuições de V. pusilla (Wagner, 1843) e $V$. thyone Thomas, 1909 - anteriormente consideradas uma mesma espécie (Koopman 1993, Nogueira et al. 1999, Lim et al. 2003). Vampyressa pusilla ocorre no sul do Paraguai e no sul e sudeste do Brasil, ao passo que $V$. thyone ocorre desde o sul da Bolívia e do Peru até o México (Lim \& Engstrom 2001, Lim et al. 2003). Entretanto, devido à insuficiência de inventários e à raridade das espécies, mais estudos são necessários para confirmar a alopatria entre estas espécies de Vampyressa. O objetivo desta nota é reportar a ocorrência de $V$. pusilla na região sul da planície do Pantanal, assim como no planalto ao seu entorno.

\section{Material e Métodos}

O Pantanal é uma extensa planície inundável, de clima tropical quente com inverno seco (Aw de Köppen). A pluviosidade média anual é de aproximadamente $1100 \mathrm{~mm}$, com chuvas concentradas entre os meses de novembro e março. A paisagem é determinada principalmente pelos pulsos de inundação (Adámoli 1986). A vegetação é composta por campos de gramíneas, formações florestais e vegetação aquática, formando um mosaico de ambientes (Prance \& Schaller 1982). São comuns lagoas temporárias ou permanentes, denominadas "baías", circundadas por extensos cordões florestais, denominados "cordilheiras", além de matas ciliares e ilhas $(0,2$ a 4 ha) de floresta semidecídua, denominadas "capões", circundadas por campos sazonalmente inundáveis (Damasceno et al. 1999, Araujo \& Sazima 2003).

Para o estudo de morcegos foram utilizadas redes-neblina para capturas em três regiões: nascente do rio Negro (19 $46^{\circ} 30^{\prime \prime} \mathrm{S}$ e

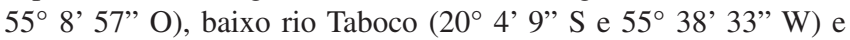

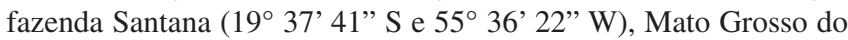
Sul. A região da nascente do Negro está situada no planalto (ca. $350 \mathrm{~m}$ altitude) de entorno do Pantanal; a região do baixo Taboco (ca. $170 \mathrm{~m}$ altitude) em transição planalto-planície e a região da fazenda Santana (ca. $115 \mathrm{~m}$ altitude) na planície. Foram realizadas duas expedições de campo para as três regiões, a primeira entre 20 de agosto e 2 de setembro de 2005 e a segunda entre 25 de janeiro e 7 de fevereiro de 2006. Foram realizadas seis noites de capturas na região do Taboco, oito noites na região da nascente do Negro e oito na região da fazenda Santana, totalizando 22 noites igualmente distribuídas entre as duas expedições. A cada noite, quatro redes de $12 \times 2,6 \mathrm{~m}$ foram mantidas abertas durante seis horas entre 18:00 e 01:00 horas. Para cada morcego capturado foram tomadas medidas de massa, com dinamômetro portátil $( \pm 0,1 \mathrm{~g})$, e de comprimento do antebraço, com paquímetro $( \pm 0,1 \mathrm{~mm})$, bem como registrados o sexo e o estado reprodutivo. Os morcegos foram então marcados com anilhas numeradas e soltos, exceto exemplares coletados para confirmação da identificação (cf. Vizotto \& Taddei 1973). Medidas adicionais foram tomadas de dois espécimes coletados de V. pusilla: comprimento do crânio, largura do arco zigomático, largura interorbital, largura do mastóide, maior largura entre os molares superiores, comprimento maxilar e comprimento mandibular. Os exemplares de V. pusilla foram incluídos na Coleção Zoológica de Referência da Universidade Federal de Mato Grosso do Sul (nos. IT056 e IT254). A identificação de V. pusilla foi baseada em Lewis \& Wilson (1987) e Lim et al. (2003).

\section{Resultados e Discussão}

Foram encontrados cinco indivíduos de Vampyressa pusilla dentre 478 morcegos capturados, de 19 espécies. Exclusivamente em ambientes de cordilheiras e matas ciliares, onde V. pusilla foi registrada, foram capturados 226 indivíduos de 17 espécies. Esta baixa proporção de V. pusilla registrada em toda a amostra (5/478) ou mesmo apenas nos ambientes onde ocorreu (5/226) indica que a espécie é rara nos locais de estudo, assim como reportado para outras regiões (Lewis \& Wilson 1987, Miretzki 2003). Os indivíduos de V. pusilla foram capturados em duas regiões. Na região da nascente do rio Negro, em mata ciliar, foram capturados três indivíduos, sendo uma fêmea adulta (21:18 horas) em 21 de agosto de 2005 (coletada) e uma fêmea grávida (20:25 horas) e um macho adulto (20:15 horas) em 27 de janeiro de 2006 (soltos) (Figura 1). Na fazenda Santana, em cordilheira, foram capturados dois machos adultos (21:30 e 23:00 horas) em 31 de janeiro de 2006, sendo um deles coletado e o outro solto. No baixo Taboco, região de transição entre a planície e o planalto, V. pusilla não foi registrada. A ocorrência em matas ciliares tem sido descrita para V. pusilla em outras regiões, e associada à disponibilidade de figueiras neste tipo de ambiente (Bonaccorso 1979, Pedro et al. 1997, Nogueira \& Perachi 2002, Bordignon 2006). Uma vez que figueiras também são muito freqüentes em cordilheiras (Pott \& Pott 2001), este fato poderia explicar parcialmente a ocorrência de $V$. pusilla registrada na fazenda Santana.

As medidas tomadas dos exemplares de $V$. pusilla registrados neste estudo foram semelhantes às esperadas para esta espécie e superiores às reportadas para $V$. thyone (Tabela 1). Dentre as nove medidas registradas, sete - massa, comprimento do antebraço, comprimento do crânio, largura interorbital, largura entre molares, comprimento maxilar e comprimento mandibular - apresentaram valores mínimos e máximos levemente superiores para exemplares capturados no presente estudo que para aqueles reportados por Lim et al. (2003), indicando que indivíduos de V. pusilla nos locais de estudo podem ser ligeiramente maiores que os de outras regiões. Entretanto, duas medidas - largura do arco zigomático e largura do mastóide - apresentaram valores levemente inferiores àqueles previamente reportados para V. pusilla (Tabela 1).

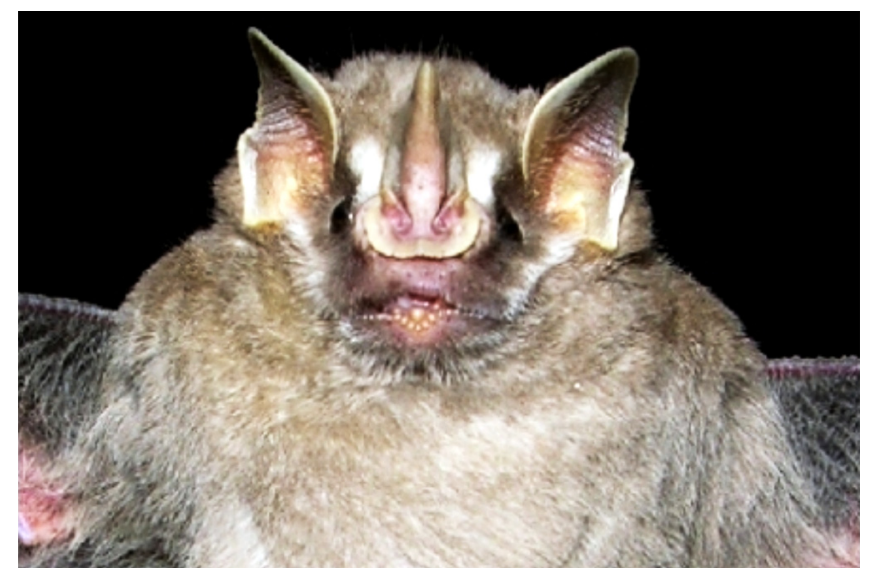

Figura 1. Macho adulto de Vampyressa pusilla (Phyllostomidae) (foto: Ana Rodrigues).

Figure 1. Adult male of Vampyressa pusilla (Phyllostomidae) (photograph: Ana Rodrigues). 
Os registros de $V$. pusilla nas regiões da nascente do rio Negro e da planície pantaneira (presente estudo) ampliam os limites de distribuição desta espécie em direção ao limite sul da distribuição de V. thyone, reduzindo em aproximadamente $400 \mathrm{~km}$ a distância que separa as distribuições das duas espécies (Figura 2) (q.v. Lim et al. 2003 para discussão sobre distância entre os limites de distribuição das espécies). A ocorrência de $V$. pusilla em cordilheiras (presente estudo) gera expectativa de que esta espécie pode ocorrer em toda a planície, destacando a importância de novos inventários nas regiões central e norte do Pantanal. Caso seja confirmada ampla ocorrência de V. pusilla no Pantanal, esta formação poderia ser considerada região de ligação entre as distribuições de $V$. pusilla e $V$. thyone,

Tabela 1. Amplitude de variação de nove medidas tomadas de dois a cinco exemplares de Vampyressa pusilla (Phyllostomidae) capturados no Pantanal e planalto de entorno (presente estudo), e de 10 exemplares de V. pusilla e 31 de V. thyone registrados por Lim et al. (2003).

Table 1. Range of variation of nine measurements taken from two to five Vampyressa pusilla (Phyllostomidae) specimens captured in the Pantanal and in the neighboring uplands (this study), and from $10 \mathrm{~V}$. pusilla and $31 \mathrm{~V}$. thyone recorded by Lim et al. (2003).

\begin{tabular}{|c|c|c|c|}
\hline \multirow[t]{2}{*}{ Caracteres } & \multirow{2}{*}{$\begin{array}{c}\text { Presente estudo } \\
V \cdot \text { pusilla }\end{array}$} & \multicolumn{2}{|c|}{ Lim et al. (2003) } \\
\hline & & V. pusilla & V. thyone \\
\hline Massa (g) & $15-18,3$ & $12-18,1$ & $8,2-11,3$ \\
\hline Comprimento do antebraço (mm) & $35-40$ & $33-36$ & $30-34$ \\
\hline Maior comprimento do crânio & $20,1-22$ & $19,5-20,6$ & $17,8-18,8$ \\
\hline Largura do arco zigomático & $11,2-11,3$ & $11,2-12,1$ & $10,2-11,1$ \\
\hline Largura interorbital & $5,1-5,7$ & $4,8-5,3$ & $4,4-5,1$ \\
\hline Largura do mastóide & $9,3-9,7$ & $9,5-9,9$ & $8,8-9,8$ \\
\hline Maior largura através dos molares superiores & $8,3-9$ & $8,1-8,8$ & $7,2-8,1$ \\
\hline Comprimento maxilar na linha da dentição & $6,9-7,5$ & $6,6-7,3$ & $5,8-6,6$ \\
\hline Comprimento mandibular na linha da dentição & $6,8-7,1$ & $6,4-6,9$ & $5,5-6,1$ \\
\hline
\end{tabular}

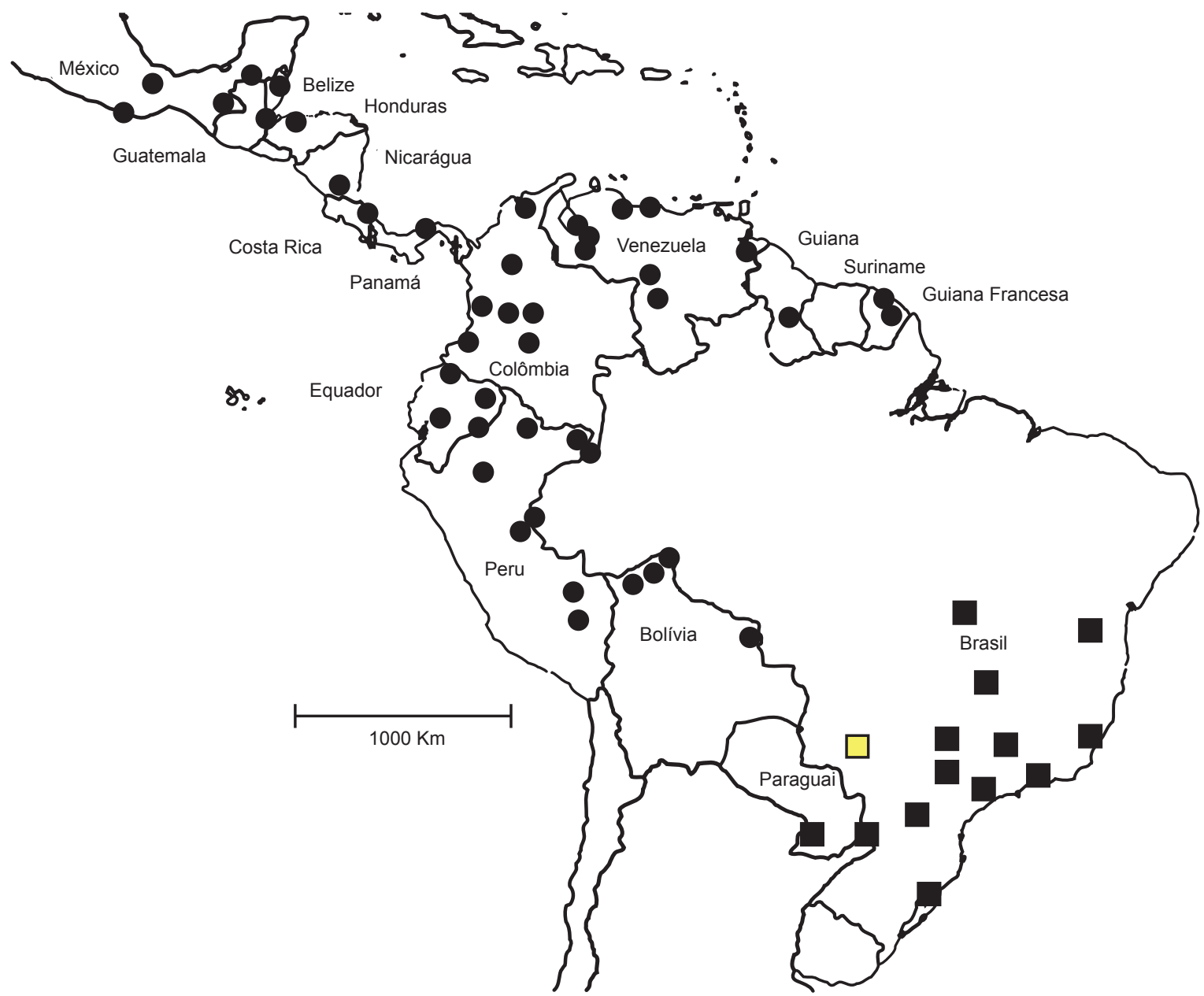

Figura 2. Distribuição de Vampyressa pusilla (Phyllostomidae) (quadrados pretos) e V. thyone (círculos), modificado de Lim et al. (2003), e localização dos registros de $V$. pusilla no presente estudo (quadrado amarelo).

Figure 2. Distribution of Vampyressa pusilla (Phyllostomidae) (black squares) and V. thyone (circles), modified from Lim et al. (2003), and location of V. pusilla records in this study (yellow square). 
em oposição à idéia atual de que representa região de disjunção (Pedro et al. 1997, Lim et al. 2003). Adicionalmente, os registros de $V$. pusilla no Pantanal ampliam para 62 o número de espécies de morcegos listadas para este ecossistema (Marinho-Filho \& Sazima 1998, Camargo \& Fischer 2005).

\section{Agradecimentos}

À Ana Rodrigues, Alan Eriksson, Roberto Munin, Luis Felipe Carvalho, Elaine Pinto e Guilli Silveira pelo valioso auxílio no campo; à Renata Pardini e um revisor anônimo pelas sugestões feitas sobre a primeira versão do manuscrito; à Conservação Internacional do Brasil, Earthwatch Institute, Fundação Manoel de Barros e Fundect-MS pelo apoio logístico e financiamento.

\section{Referências Bibliográficas}

ADÁMOLI, J.A. 1986. A dinâmica de inundações no Pantanal. In Anais do I Simpósio sobre Recursos Naturais e Socioeconômicos do Pantanal. CPAP Embrapa, Corumbá, p. 51-62.

ARAUJO, A.C. \& SAZIMA, M. 2003. The assemblage of flowers visited by hummingbirds in the "capões" of Southern Pantanal, Mato Grosso do Sul, Brazil. Flora 198:427-435.

BONACCORSO, F.J. 1979. Foraging and repoductive ecology in a Panamanian bat community. Bull. Florida State Mus, Biol. Sci. 24:359-408.

BORDIGNON, M.O. 2006. Diversidade de morcegos (Mammalia, Chiroptera) do Complexo Aporé-Sucuriú, Mato Grosso do Sul, Brasil. Revista Brasileira de Zoologia 23(4):1002-1009.

CAMARGO, G. \& FISCHER, E. 2005. Primeiro registro do morcego Mimon crenulatum (Phyllostomidae) no Pantanal, sudoeste do Brasil. Biota Neotropica 5(1) - http://www.biotaneotropica.org.br/v5n1/pt/ abstract?short-communication+BN00705012005. ISSN 1676-0603.

DAMASCENO, G.A., BEZERRA, M.A.O., BORTOLOTTO, I. \& POTT, A. 1999. Aspectos florísticos e fitofisionômicos dos capões do Pantanal do Abobral. In: Anais do II Simpósio sobre Recursos Naturais e Sócio-econômicos do Pantanal - Manejo e Conservação. CPAP Embrapa \& UFMS, Corumbá, p. 203-214.
KOOPMAN, K.F. 1993. Order Chiroptera. p. 137-241 in Mammal Species of the World: a taxonomic and geographic reference (D. E. Wilson and D. M. Reeder, eds.). Smithsonian Institution Press. Washington.

LEWIS, S.E. \& WILSON, D.E. 1987. Vampyressa pusilla. Mammalian Species. 292:1-5.

LIM, B.K., \& ENGSTROM, M.D. 2001. Species diversity of bats (Mammalia: Chiroptera) in Iwokrama Forest, Guyana, and the Guianan subregion: implications for conservation. Biodiversity and Conservation, 10:613-657.

LIM, B.K., PEDRO, W.A., \& PASSOS, F.C. 2003. Differentiation and species status of the Neotropical yellow-eared bats Vampyressa pusilla and $V$. thyone (Phyllostomidae) with a molecular phylogeny and review of the genus Acta Chiropterologica, 5(1):15-29.

MARINHO-FILHO, J. \& SAZIMA, I. 1998. Brazilian bats and conservation. In Bat Biology and Conservation (T. H. Kunz and P. Racey, eds.). Smithsonian Institution Press, p. 282-294.

MIRETZKI, M. 2003. Morcegos do Estado do Paraná, Brasil (Mammalia, Chiroptera): Riqueza de espécies, distribuição e síntese do conhecimento atual. Papéis Avulsos de Zoología, 43(6):101-138.

NOGUEIRA, M. R., POL, A. \& PERACCHI, A. L. 1999. New records of bats from Brazil with a list of additional species for the chiropteran fauna of the state of Acre, western Amazon basin. Mammalia, 63: 363-368.

NOGUEIRA, M.R. \& PERACCHI, A.L. 2002. Fig-seed predation by 2 species of Chiroderma: discovery of a new feeding strategy in bats. Journal of Mammalogy, Lawrence, 84(1):225-233.

POTT, A \& POTT, V.J. 1994. Plantas do Pantanal. Brasília: EMBRAPA SPI. 320p.

PEDRO, W.A., CARVALHO, C., HAYASHI, M.M., BREDT, A., ARMANI, N.M.S., SILVA M.M.S., GOMES, L., GONÇALVES, C.A. \& PERES, N.F. 1997. Notes on Vampyressa pusilla (Wagner, 1843) in the south of São Paulo State. Chiroptera Neotropical, 3(2):79-80.

PRANCE, G.T. \& SCHALLER, G.B. 1982. Preliminary study of some vegetation types of the Pantanal, Mato Grosso, Brazil. Brittonia 34:228-251.

VIZOTTO, L.D. \& TADDEI, V.A. 1973. Chave para determinação de quirópteros brasileiros. Revista da Faculdade de Filosofia, Ciências e Letras de São José do Rio Preto, Boletim de Ciências, v. 1, p. 1-72. 